\title{
Withaferin A Inhibits the Proteasome Activity in Mesothelioma In Vitro and In Vivo
}

\author{
Huanjie Yang 2,69 , Ying Wang ${ }^{29}$, Vino T. Cheryan ${ }^{1,3}$, Wenjuan $\mathrm{Wu}^{1,3}$, Cindy Qiuzhi Cui ${ }^{2}$, Lisa A. Polin ${ }^{3}$, \\ Harvey I. Pass ${ }^{2,5}$, Q. Ping Dou ${ }^{2,4 *}$, Arun K. Rishi ${ }^{1,2,3 *}$, Anil Wali ${ }^{1,2 \times}$
}

1 John D. Dingell VA Medical Center, Wayne State University, Detroit, Michigan, United States of America, 2 Karmanos Cancer Institute, Wayne State University, Detroit, Michigan, United States of America, 3 Departments of Oncology, Wayne State University, Detroit, Michigan, United States of America, 4 Department of Pathology, Wayne State University, Detroit, Michigan, United States of America, $\mathbf{5}$ New York University Cancer Center, New York, New York, United States of America, $\mathbf{6}$ Department of Life Science and Engineering, Harbin Institute of Technology, Harbin, China

\begin{abstract}
The medicinal plant Withania somnifera has been used for over centuries in Indian Ayurvedic Medicine to treat a wide spectrum of disorders. Withaferin A (WA), a bioactive compound that is isolated from this plant, has anti-inflammatory, immuno-modulatory, anti-angiogenic, and anti-cancer properties. Here we investigated malignant pleural mesothelioma (MPM) suppressive effects of WA and the molecular mechanisms involved. WA inhibited growth of the murine as well as patient-derived MPM cells in part by decreasing the chymotryptic activity of the proteasome that resulted in increased levels of ubiquitinated proteins and pro-apoptotic proteasome target proteins ( $221, B a x, I \kappa B \alpha)$. WA suppression of MPM growth also involved elevated apoptosis as evidenced by activation of pro-apoptotic p38 stress activated protein kinase (SAPK) and caspase-3, elevated levels of pro-apoptotic Bax protein and cleavage of poly-(ADP-ribose)-polymerase (PARP). Our studies including gene-array based analyses further revealed that WA suppressed a number of cell growth and metastasis-promoting genes including c-myc. WA treatments also stimulated expression of the cell cycle and apoptosis regulatory protein (CARP)-1/CCAR1, a novel transducer of cell growth signaling. Knock-down of CARP-1, on the other hand, interfered with MPM growth inhibitory effects of WA. Intra-peritoneal administration of $5 \mathrm{mg} / \mathrm{kg}$ WA daily inhibited growth of murine MPM cell-derived tumors in vivo in part by inhibiting proteasome activity and stimulating apoptosis. Together our in vitro and in vivo studies suggest that WA suppresses MPM growth by targeting multiple pathways that include blockage of proteasome activity and stimulation of apoptosis, and thus holds promise as an anti-MPM agent.
\end{abstract}

Citation: Yang H, Wang Y, Cheryan VT, Wu W, Cui CQ, et al. (2012) Withaferin A Inhibits the Proteasome Activity in Mesothelioma In Vitro and In Vivo. PLoS ONE 7(8): e41214. doi:10.1371/journal.pone.0041214

Editor: Sharmila Shankar, University of Kansas Medical Center, United States of America

Received April 16, 2012; Accepted June 24, 2012; Published August 17, 2012

This is an open-access article, free of all copyright, and may be freely reproduced, distributed, transmitted, modified, built upon, or otherwise used by anyone for any lawful purpose. The work is made available under the Creative Commons CCO public domain dedication.

Funding: This work was supported by the Department of Veterans Affairs Merit Review grants (AKR and AW), Mesothelioma Applied Research Foundation (MARF) support (AW), and National Cancer Institute Grants CA120009, CA112625 (QPD), and the National Cancer Institute at the National Institutes of Health (NCI/ $\mathrm{NIH}$ ) Cancer Center Support Grant to Karmanos Cancer Institute. The funders had no role in study design, data collection and analysis, decision to publish, or preparation of the manuscript.

Competing Interests: The authors have declared that no competing interests exist.

*E-mail: doup@karmanos.org (QPD); Rishia@Karmanos.org (AKR)

9 These authors contributed equally to this work.

a Current address: Center to Reduce Cancer Health Disparities, National Cancer Institute, National Institutes of Health, Rockville, Maryland, United States of America

\section{Introduction}

Malignant pleural mesothelioma (MPM) is a lethal asbestosrelated malignancy [1]. Despite aggressive multimodality treatment involving surgery, adjuvant or neoadjuvant chemotherapy, and radiation [2], the median survival of MPM is about 9-17 months [3]. Millions of American workers have been exposed to asbestos, and exposure to asbestos has been shown to increase the risk of several serious diseases including asbestosis, lung cancer and mesothelioma [1]. It is estimated that there are 2,000 to 3,000 people diagnosed as MPM patients each year in the United States and the incidence of this disease is expected to increase in the next decade in United States and Europe [3,4]. Due to the resistance to currently available chemotherapies and the increasing incidence of MPM, development of new treatments for MPM is urgently needed.
A number of studies suggest that agents derived from plants including dietary fruits and vegetables are helpful in either inhibiting or reversing the development of cancer [5-7]. A medicinal plant, Withania somnifera, is commonly used in Indian traditional medicine to treat a range of disorders [8]. Withaferin-A (WA), a steroidal lectone is a bioactive compound isolated from Withania somnifera, exhibits anti-inflammatory, immunomodulatory, and anti-angiogenic properties [9,10]. WA suppresses growth of human cancer cells of prostate, breast, and soft tissue sarcoma origins in vitro and in vivo [11-13]. Although the mechanisms of action of WA have yet to be fully defined, the pro-survival NF- $\kappa \mathrm{B}$ and AKT, pro-apoptotic Par-4, FOXO-3, and Bim, and the proteasomal chymotrypsin subunit $\beta 5$ have been shown to be molecular targets of WA signaling [11,14-16]. WA treatments cause alterations in the cytoskeletal architecture, induce generation of reactive oxygen species, mitochondrial dysfunction and proteasomal inhibition $[14,17,18]$. 
Here we investigated effects of WA on growth of MPM cells in vitro. Consistent with observations in other models, WA caused cell growth inhibition that involved diminished proteasomal activities and elevated apoptosis. Although WA stimulated expression of pro-apoptotic Bax, and activated p38 SAPK and caspase-3/7, our gene-array-based analysis revealed that WA suppressed expression of a number of cell growth and metastasis transducers. Moreover, WA suppression of MPM cell growth involved stimulation of a novel transducer of cell growth and apoptosis signaling CARP-1/ CCAR1 [19-21]. Intra-peritoneal administration of WA suppressed growth of murine mesothelioma allografts in part by enhancing apoptosis. Our proof-of-concept studies reveal, for the first time, MPM inhibitory properties of WA and are expected to facilitate utilization of this agent or its potent derivatives as potential adjuvants for treatment and perhaps chemoprevention of MPM.

\section{Methods}

\section{Cells and Reagents}

MPM patient derived cell lines [H2373, H2452, H2461, and H2595] established in our laboratory and characterized in detail [22] were cultured in RPMI 1640 supplemented with 100 units/ $\mathrm{ml}$ of penicillin, $100 \mu \mathrm{g} / \mathrm{ml}$ streptomycin, $4 \mathrm{mM} \mathrm{L}$-glutamine, and $10 \%$ fetal calf serum. H226 MPM cells were obtained from ATCC (Manassas, VA) and maintained following vendor's guidelines. The AB12 murine malignant mesothelioma cell line was derived from BALB/c mice and was shown to form subcutaneous tumors when implanted in mice $[23,24]$. This cell line was cultured and maintained in high-glucose DMEM supplemented with $10 \%$ fetal bovine serum (FBS), 100 units $/ \mathrm{mL}$ penicillin, $100 \mu \mathrm{g} / \mathrm{mL}$ streptomycin. FBS was obtained from Tissue Culture Biologicals (Tulare, CA). RPMI 1640 medium, penicillin and streptomycin were purchased from Invitrogen Co. (Carlsbad, CA). DMEM was purchased from Mediatech Inc (Herndon, VA). Cells were incubated at $37^{\circ} \mathrm{C}$ in a humidified atmosphere of $5 \% \mathrm{CO}_{2}$ in air and were passaged weekly. Purified withaferin A $(>98 \%)$ was purchased from ChromaDex, Inc. (Santa Ana, CA) and dissolved in dimethyl sulfoxide (DMSO; Sigma; St. Louis, MO) at a stock concentration of $50 \mathrm{mM}$, aliquoted and stored at $-20^{\circ} \mathrm{C}$. Purified rabbit $20 S$ proteasome, mouse monoclonal antibody p21, fluorogenic substrates N-Succinyl-Leu-Leu-Val-Tyr-7-amino-4-methylcoumarin (Suc-LLVY-AMC) for the proteasomal chymotryptic activity and the caspase-3/-7-specific substrate N-acetyl-Asp-GluVal-Asp-7-amino-4-methylcoumarin (Ac-DEVD-AMC) were obtained from Calbiochem Inc. (San Diego, CA). Anti-PARP mouse monoclonal antibody was purchased from BIOMOL International LP (Plymouth Meeting, PA). Anti-Bax (B-9), anti-p27 (F-8), anti-cmyc (9E10), and anti-Ubiquitn (P4D1) mouse monoclonal antibodies as well as anti-inhibitor of nuclear factor $\kappa \mathrm{B}-\alpha(\mathrm{I} \kappa \mathrm{B}-\alpha)$ (C-15), anti-c-Jun (H-79), anti-vimentin (V9) rabbit polyclonal, and anti-actin (C-11) goat polyclonal antibodies were obtained from Santa Cruz Biotechnology Inc. (Santa Cruz, CA). Mouse monoclonal antibody NCL-p27 was purchased from Novocastra Laboratories Ltd (Newcastle upon Tyne, UK). Anti-p38 and phospho-p38 rabbit polyclonal antibodies were obtained from Cell Signaling (Beverly, MA). Generation and characterization of the anti-CARP-1/CGAR1 rabbit polyclonal antibodies have been described before [19]. Enhanced Chemiluminescence Reagent was purchased from Amersham Biosciences (Piscataway, NJ) and the Apoptag Peroxidase in situ Apoptosis Detection Kit was obtained from Chemicon International, Inc. (Temecula, CA). Protein Assay Kit was purchased from Bio-Rad Laboratories (Hercules, CA), while 3-4, 5-dimethyltiazol-2-yl-2.5-diphenyl- tetrazolium bromide (MTT), cremophor and other chemicals were obtained from Sigma-Aldrich (St. Louis, MO). The ONTarget plus SiRNAs for knock-down of CARP-1 and DharmaFECT transfection reagent for Si-RNA transfections were purchased from Dharmacon Inc., Thermo Fisher Scientific (Lafayette, GO).

\section{Cell Growth Inhibition Studies by MTT Assay}

MPM (H2373, H2452, H2461, H226 and AB12) cells $\left(5 \times 10^{3}\right)$ were seeded in a 96-well culture plate and subsequently treated with WA at different concentrations for noted times. Control cells were treated with $0.1 \%$ DMSO in culture medium. After treatment, the cells were incubated with $1 \mathrm{mg} / \mathrm{ml}$ of MTT reagent at $37^{\circ} \mathrm{C}$ for $4 \mathrm{~h}$ and then MTT was removed and $100 \mu \mathrm{L}$ of DMSO was added, followed by colorimetric analysis using a multilabel plate reader at $560 \mathrm{~nm}\left(\right.$ Victor $^{3}$; PerkinElmer, Wellesley, MA, USA).

\section{Inhibition of cellular 265 proteasome activity}

MPM cells were treated with either DMSO or WA for indicated times, followed by extraction of whole cell lysate. Proteins from whole cell lysate were incubated with the proteasomal chymotrypsin-like specific substrate Suc-LLVY-AMC (at $20 \mu \mathrm{M}$ ). The proteasomal activity was measured by hydrolysis of their substrates, with 355-nm excitation and 460-nm emission wavelengths.

\section{Cell-free Caspase-3/-7 activity assay}

MPM cells were treated with different concentrations of WA for indicated time periods. The prepared whole cell extract (30 $\mu \mathrm{g}$ per sample) was then incubated with $40 \mu \mathrm{M}$ of caspase-3/-7 substrate Ac-DEVD-AMC in $100 \mu$ of the assay buffer $(20 \mathrm{mM}$ Tris- $\mathrm{HCl}$, $\mathrm{pH} 7.5)$ at $37^{\circ} \mathrm{C}$ for at least $2 \mathrm{~h}$. The release of the AMC groups was measured as above.

\section{Western blot analysis}

Cells were harvested and lysed in RIPA buffer $(50 \mathrm{mM}$ TrisHCI, pH 8.0, $150 \mathrm{mM}$ sodium chloride, $1.0 \%$ NP-40, $0.5 \%$ sodium deoxycholate, $0.1 \%$ sodium dodecyl sulfate (SDS), and $0.1 \%$ of protease inhibitor cocktail) for $20 \mathrm{~min}$ at $4{ }^{\circ} \mathrm{C}$. The lysates were centrifuged at $14,000 \mathrm{rpm}$ at $4^{\circ} \mathrm{C}$ for $15 \mathrm{~min}$ to remove debris. Protein concentrations of whole cell lysates were determined using the Protein Assay Kit. Supernatant proteins, $50 \mu \mathrm{g}$ from each sample, were separated by SDS- $10 \%$ polyacrylamide gel electrophoresis (SDS-PAGE) and transferred to polyvinylidene difluoride (PVDF) membrane (Bio-rad, Hercules, CA) by standard procedures. The membranes were hybridized with primary antibodies followed by incubation with appropriate secondary antibodies. The antibody-bound proteins were visualized by treatment with the chemiluminescence detection reagent (Pierce) according to manufacturer's instructions, followed by exposure to film (Kodak X-Omat). The same membrane was re-probed with the anti- $\beta$ actin antibody, which was used as an internal control for protein loading.

\section{Isolation of RNA and microarray analysis}

Total RNA was extracted from untreated or WA-treated H2373 MPM cells. At the end of treatments, the untreated and treated cells were harvested and total RNA were isolated, and purified using the RNeasy Mini kit and RNase-free DNase Set (Qiagen, Valencia, CA) according to the manufacturer's protocols. WAdependent changes in gene expression in MPM cells were performed at the Genomic Core Facility, Karmanos Cancer 
Institute utilizing Illumina BeadChip ${ }^{\circledR}$ Arrays essentially according to manufacturer's instruction (Illumina). Briefly, $0.5 \mu \mathrm{g}$ total RNA was biotin-labeled and hybridized with BeadChips. The signal was detected with streptovadin-Cy3 according to manufacturer's instruction (Illumina). The imaging of the BeadChips was conducted using a Bead Array Reader in conjunction with Bead Studio software (Illumina). Normalization of the data was carried out using a quantile based approach which transforms the raw data so that the resulting normalized expression values of each sample have the same distribution [25]. An unsupervised cluster analysis was performed to detect similarities among samples based on gene expression profiles. The genes retained to perform the clustering were those varying the most regardless their group membership as described elsewhere [26]. Significance of the differentially expressed genes among various groups was tested using a moderated $t$-test to allow for $p$-value computation for the significance of gene changes. The $p$-values were then adjusted using the False Discovery Rate method [26] to derive corrected $p$ values. The $p$ values of $<0.5$ were considered significant provided that the fold change in expression was also equal to or larger than 2 .

\section{Murine mesothelioma allograft experiments}

All animal work was done in accordance with protocols approved by the Institutional Laboratory Animal Care and Use Committee of Wayne State University. Female BALB/c mice aged 5 weeks were purchased from Taconic Research Animal Services and housed in accordance with protocols approved by the WSU IACUC. On day 1 AB12 murine mesothelioma cells $\left(0.5 \times 10^{6}\right)$ were suspended in $0.1 \mathrm{~mL}$ serum-free DMEM medium and inoculated subcutaneously (s.c.) in the right flank of each mouse ( $\mathrm{n}=5$ each group). When the tumors became palpable (on day 10 after inoculation), the mice were randomly assigned into 2 groups. The control group animals received a daily i.p. injection with 50$100 \mu \mathrm{l}$ of a vehicle [10\% DMSO, 70\% Cremophor:ethanol (3:1) and 20\% PBS] while the test group animals received 50-100 $\mu \mathrm{l}$ of $5.0 \mathrm{mg} / \mathrm{kg}$ WA daily. Tumor sizes were measured daily using calipers and their volumes calculated using a standard formula: width ${ }^{2} \times$ length $/ 2$. Body weight was measured weekly. The mice were sacrificed after 16 day-treatment when control tumors reached to $\sim 1774 \mathrm{~mm}^{3}$. H\&E staining confirmed the presence of tumor.

\section{Immunohistochemical analysis}

Apoptosis in tumor tissues was determined by TUNEL assay using in situ cell Death Detection kit from Roche Applied Science (Indianapolis, IN) according to the manufacturer's instruction. The formalin-fixed tumor xenograft biopsies from untreated or WA-treated animals were paraffin embedded and processed essentially following our previously described procedures [27]. The tumor tissue slides were stained for presence of CARP-1, cyclin-dependent kinase inhibitor p27, and oncogene c-myc by utilizing respective antibodies, and were then photographed under different magnifications using Zeiss microscope with a $35 \mathrm{~mm}$ camera attached for recording the photomicrographs. $\mathrm{H} \& \mathrm{E}$ counter-staining of tumor tissues was performed following our previously described methods [27]. The proteasomal or caspase activity assays and Western blotting using animal tumor tissue samples were performed similarly as described above using cultured MPM cells.

\section{Results}

\section{WA inhibits MPM cell proliferation}

We first tested the growth-inhibitory effects of WA on four human MPM cell lines that were derived from mesothelioma patients and one murine mesothelioma cell line. WA potently inhibited cell proliferation in all tested cell lines (Fig. 1). Among them, H2373 cells were most sensitive towards WA treatment, showing $52 \%$ inhibition of their growth in the presence of $1.25 \mu \mathrm{M}$ dose (Fig. 1). H2595, H226, H2452 and AB12 cell lines were relatively less sensitive to WA treatment, exhibiting 58, 67, 55 and $55 \%$ inhibition, respectively, at $5 \mu \mathrm{M}$ dose (Fig. 1).

\section{WA inhibits proteasome activity and stimulates apoptosis in MPM cells}

WA was previously reported to target multiple molecules, such as FOX3a, or the proteasome [11,14], to suppress cell growth. Consistent with these observations, treatments of H2595 MPM cells with $10 \mu \mathrm{M}$ dose of WA for up to $24 \mathrm{~h}$ elicited about $70 \%$ inhibition of the proteasomal chymotrypsin-like activity (Fig. 2A). The proteasome inhibition was noted as early as $2 \mathrm{~h}$ of WA exposure, and remained inhibited to the same extent for the rest of the treatment periods (Fig. 2A). Similar to human MPM cells, WA also inhibited the proteasomal chymotrypsin-like activity in murine AB12 MPM cells (Fig. 2G). The chymotryptic activity of the proteasome was inhibited around $10 \%$ within $1 \mathrm{~h}$ after addition of $5 \mu \mathrm{M} \mathrm{WA}$ and further decreased by $25-30 \%$ during $2-$ $6 \mathrm{~h}$ and leading to 52 to $62 \%$ decline in proteasomal activity over 8-24 h (Fig. 2C). Western blot analysis that showed an increased level of ubiquitinated proteins after $2-24 \mathrm{~h}$ of WA treatment in both the human and murine MPM cells (Fig. 2B, D), as well as the accumulation of the proteasomal targets protein p21 and Bax proteins (Fig. 2B, D) suggesting inhibition of proteasome by WA.

Suppression of anti-apoptotic/pro-survival signaling pathway and/or activation of apoptotic pathway are one of the mechanisms that contribute to cell growth inhibition. WA treatments caused a time-dependent increase in levels of cell cycle inhibitory protein p21, pro-apoptotic protein Bax, and I $\mathrm{I} B-\alpha$, an endogenous inhibitor of anti-apoptotic NF- $\kappa \mathrm{B}$ (Figs. 2B, D, and $3 \mathrm{~A}, \mathrm{G}$ ), suggesting that WA inhibited MPM cells growth in part by causing apoptosis. Indeed, apoptosis induction was confirmed by multiple assays. First, WA treatments caused increased cleavage of PARP in MPM cells. As shown in Fig. 3A, a moderate PARP cleavage was

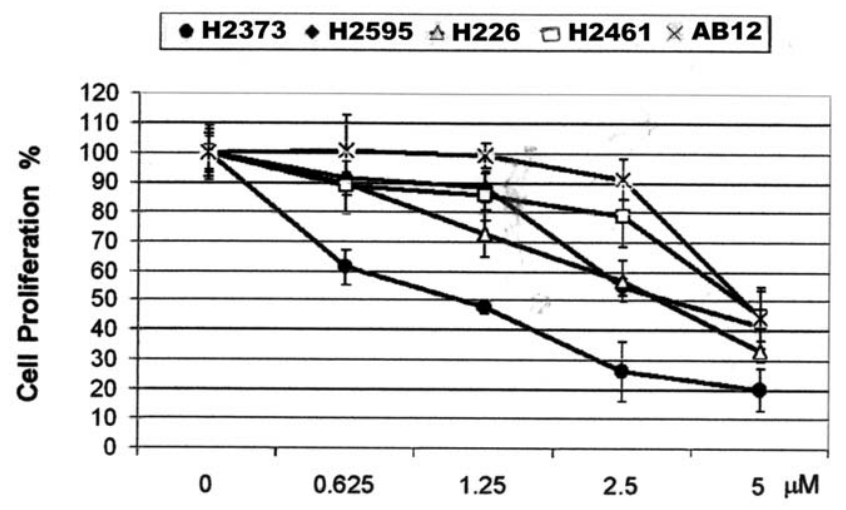

Figure 1. Antiproliferative effect of WA on human MPM cells. Cells were treated with vehicle (Control, denoted as 0 ) or indicated doses of WA for $72 \mathrm{~h}$. Determination of viable/live cells was carried out by MTT assay. The data in the histograms represent means of three independent experiments; bars, S.E.

doi:10.1371/journal.pone.0041214.g001 


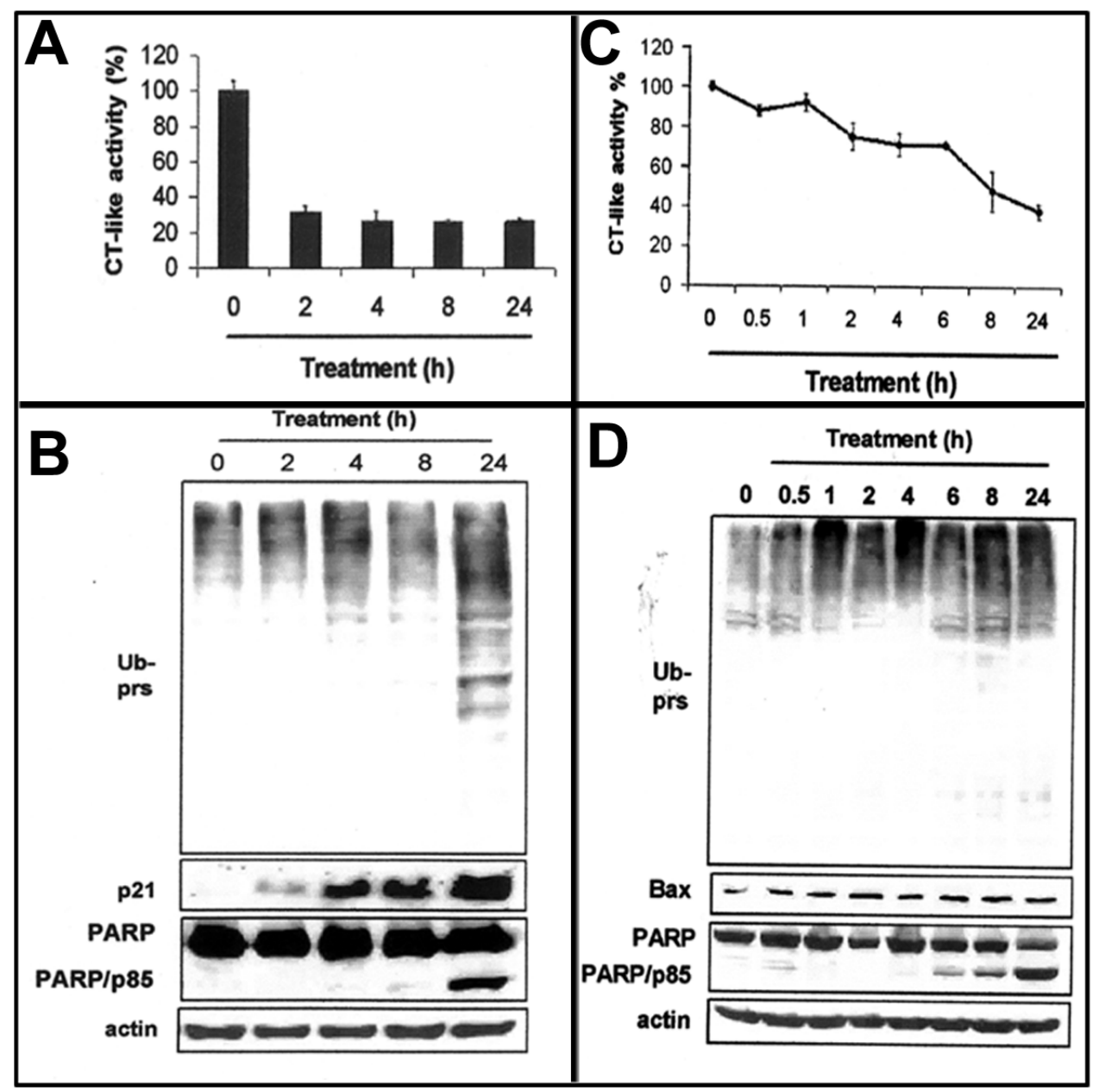

Figure 2. WA inhibits MPM cell proteasome activity. (A, B) Kinetic effects of WA on proteasome inhibition and apoptosis induction in H2595 human MPM cells. Cells were treated with $10 \mu \mathrm{M}$ WA for various times. Chymotryptic activity of the proteasome was determined as in methods (A) and accumulation of ubiquitinated proteins, levels of p21, PARP, cleaved PARP, and actin proteins were determined by Western blotting (B). (C, D) WA inhibits proteasomal chymotrypsin-like activity in AB12 murine MPM cells and induces apoptosis. Cells were treated with $10 \mu \mathrm{M}$ WA for indicated time periods, followed by measurement of proteasomal CT-like activity (C), Western blotting analysis for accumulation of ubiquitinated proteins, and levels of Bax, PARP, cleaved PARP, and actin proteins (D) as described in methods. Bars, SD; Ub prs, Ubiquitinated proteins. doi:10.1371/journal.pone.0041214.g002

observed in cells treated with $2.5 \mu \mathrm{M}$ WA, while a robust cleavage of PARP was noticeable in MPM cells treated with 5-10 $\mu \mathrm{M}$ WA (Fig. 3A). Second, a 3.6-4.7-fold increase in caspase $3 / 7$ activity was detected in 2.5-5 $\mu \mathrm{M}$ WA treated H2373 cells (Fig. 3B). WA treatments also caused caspase $3 / 7$ activation in a time-dependent manner in murine MPM cells (not shown). Finally, morphological changes associated with apoptotic nuclei were observed in $10 \mu \mathrm{M}$ WA treated cells (data not shown, but see Fig. 3E). We next studied kinetic effects of WA on apoptosis induction in H2373 cells. Levels of the pro-apoptotic protein Bax began to increase after $2 \mathrm{~h}$ of WA treatment and showed maximal increase at $24 \mathrm{~h}$ treatment (Fig. 3C). IкB- $\alpha$ levels however peaked after $2-4 \mathrm{~h}$ of WA treatment, and then dropped to the basal level at $24 \mathrm{~h}$ treatment (Fig. 3C). Increase in Bax protein in the presence of WA accompanied consistent $\sim 3$-fold activation of caspase $3 / 7$ and cleavage of PARP and apoptosis-associated morphological changes in MPM cells (Figs. 3C, D, and E).

Although, WA inhibited proteasomal activity in H2595 MPM cells within $2 \mathrm{~h}$ of treatment (Fig. 2A), the Western blotting experiments revealed cleavage of PARP at the $24 \mathrm{~h}$ treatment period (Fig. 2B). Moreover, WA treatment failed to activate caspase $3 / 7$ in H2595 cells (data not shown), suggesting that caspase 3/7-independent cleavage of PARP likely contributed to relative resistance of the $\mathrm{H} 2595$ cells to growth inhibition by WA. Taken together, our in vitro studies strongly suggest that WA suppressed growth of human and murine MPM cells by attenuating proteasomal activity and stimulating apoptosis.

\section{WA suppression of MPM growth involves novel apoptosis transducer}

The adverse side effects associated with many current anticancer therapies together with the molecular complexity of cancers are limiting factors in effective treatment/management of many cancers. For better and improved therapeutic responses it may therefore be necessary to identify additional perhaps novel cancer cell growth inhibitory targets/pathways for potential exploitation in devising efficacious therapeutic strategies. To this end, we utilized gene array-based approaches to further investigate mechanisms of MPM cell growth regulation by WA. H2373 MPM cells were either untreated or separately treated with two doses of WA as described in methods. The RNAs from each group were hybridized with gene-array chips, and the data were 
A

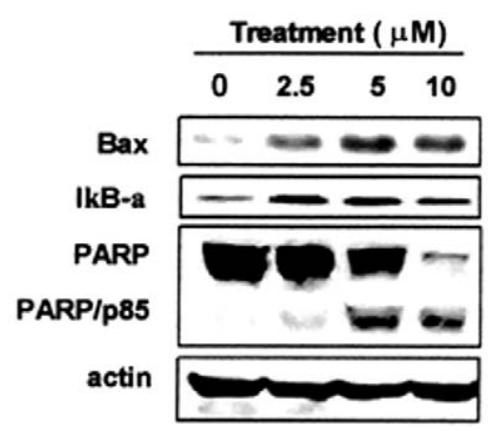

C

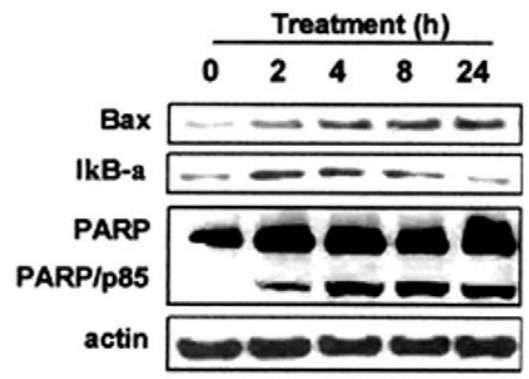

B

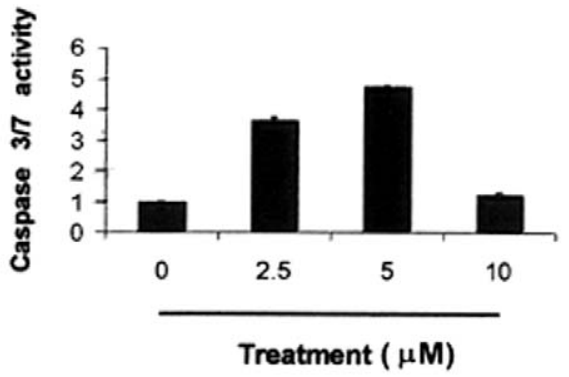

D

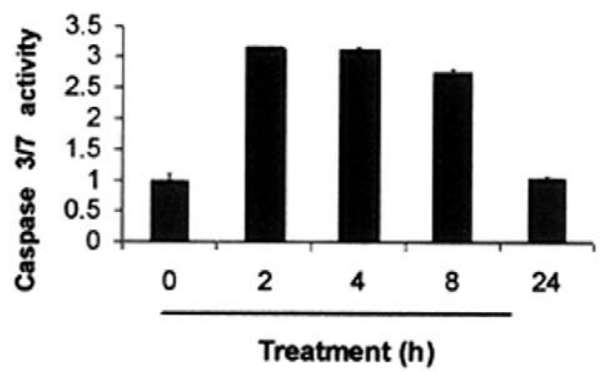

E
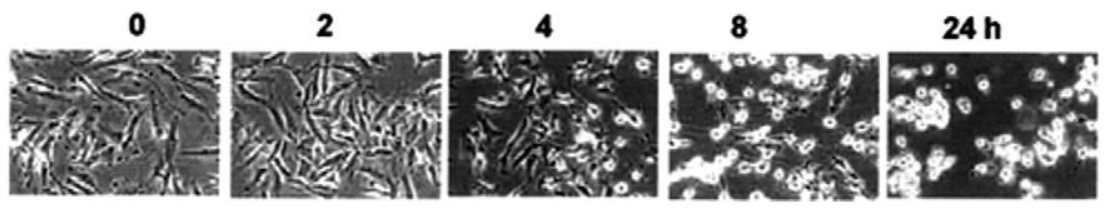

Figure 3. Dosage and kinetic effects of WA on apoptosis induction in human MPM cells. H2373 cells were treated with different concentrations of WA for $16 \mathrm{~h}(\mathrm{~A}, \mathrm{~B})$, or $10 \mu \mathrm{M}$ WA for various times (C-E), followed by Western blotting analysis for levels of Bax, IкB- $\alpha$, PARP, and actin proteins as detailed in methods (A and C). B, D, Caspase 3/7 activation was determined from by WA treated cell lysates as in methods. Bars, SD. E, Photomicrographs showing apoptosis-associated morphological changes in WA-treated MPM cells for indicated times. 0, Control, DMSO treatment. doi:10.1371/journal.pone.0041214.g003

computed to identify genes that had a significant 2-fold or higher altered expression following WA treatments. A select subset of these genes is indicated in Table 1 (see table $\mathrm{S} 1$ for complete list of WA-regulated genes in H2373 MPM cells). Interestingly, the array data revealed down-regulation of a vast majority of genes while a small number of genes were up-regulated. Among the genes that were down-regulated included cell growth and metastasispromoting oncogenes c-myc, c-fos, c-jun, while tissue inhibitor of metallopeptidases (TIMP)-2 was significantly upregulated (Table 1). Our western blot analyses further demonstrate that treatments of different human MPM cells with WA resulted in diminished levels of c-myc and c-jun proteins (Fig. 4A).

CARP-1/CCAR1 is a biphasic transducer of cell growth and apoptosis signaling [19-21,28]. With reference to the MPM model, our previous studies indicated that the proteasome inhibitor velcade as well as the chemo-preventive and dietary agent curcumin suppressed MPM cell growth in part by stimulating CARP-1 expression [5,29]. Here we investigated whether and to what extent CARP-1 was involved in MPM cell growth inhibition by WA. WA treatments of murine and human MPM cells resulted in elevated levels of CARP-1 (Fig. 4B). H2461
Cells were treated with $10 \mu \mathrm{M}$ WA for various time periods, and the cell lysates were analyzed by western blotting for CARP-1 expression. WA stimulated CARP-1 levels in a time-dependent manner with robust CARP-1 increase noticeable as early as $2 \mathrm{~h}$ of WA treatment (Fig. 4C). A number of studies have described protumorigenic and prometastatic properties of vimentin-expressing cancer cells, and vimentin expression has been found to correlate with poor outcome in many cancers [30]. Although MPM cells are mesothelial in origin and express vimentin, and the fact that WA binds to tetrameric vimentin complex [31], and targets vimentin selectively in the cancer cells [13], we investigated whether WA targeted vimentin in MPM cells. As shown in figure $4 \mathrm{C}$, WA exposure caused reduced levels of vimentin at $24 \mathrm{~h}$ of treatment. Since WA promoted apoptosis signaling and caspase 3/7 activation in MPM cells as early as $2 \mathrm{~h}$ (see Fig. 3D), and given that activated caspase- 3 is known to promote vimetin degradation [13], it is likely that WA-dependent loss of vimentin in MPM cells is in part due to its degradation by caspase-3.

Our previous studies have revealed involvement of stressactivated protein kinase (SAPK) p38 in transducing CARP-1dependent cell growth inhibitory signaling [20,21]. Because WA 


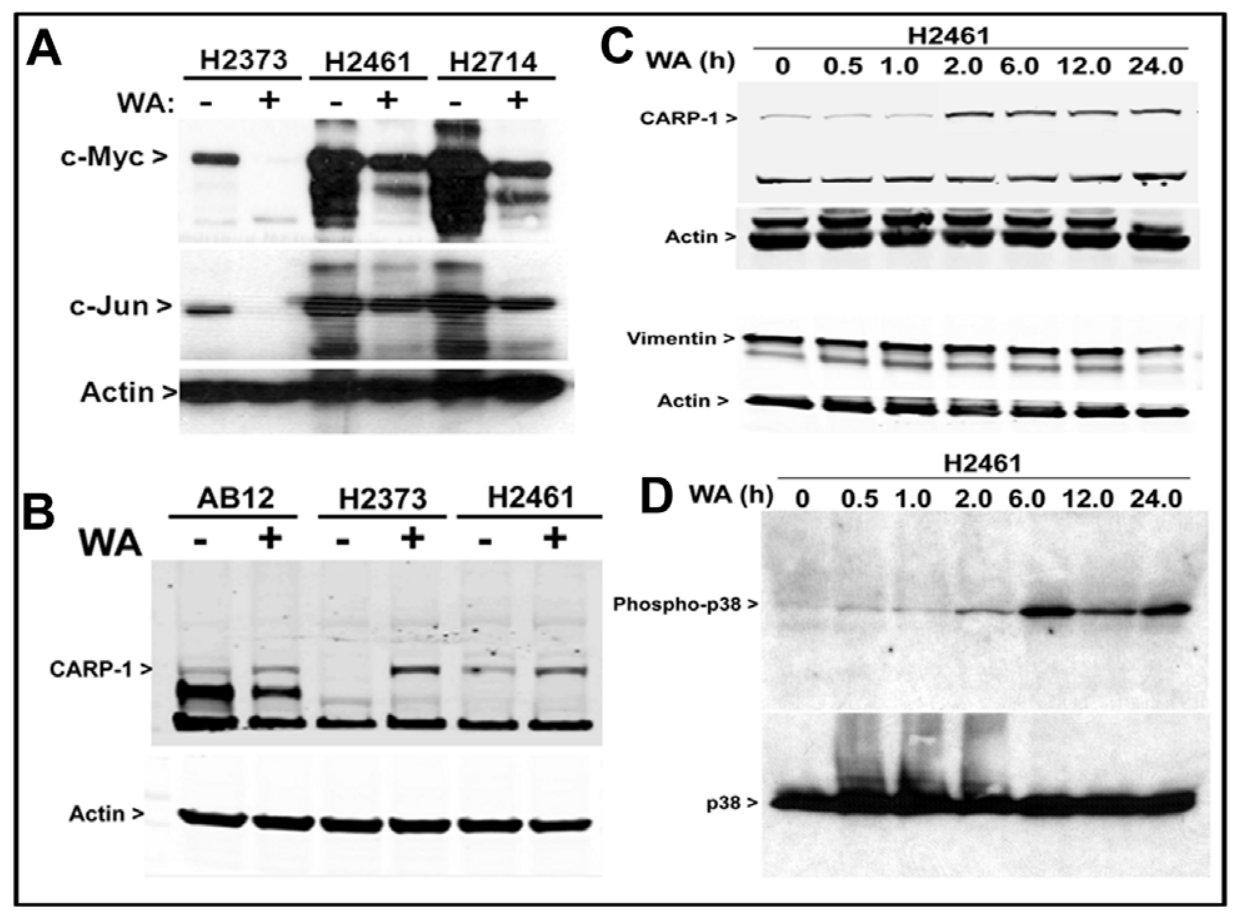

Figure 4. WA suppresses MPM survival and metastasis promoting genes while enhances expression/activation of pro-apoptotic genes. MPM cells were either untreated (DMSO; denoted as -) or treated with $10 \mu \mathrm{M}$ WA (denoted as + ) and cell lysates were analyzed by western blotting as in methods. Levels of c-myc, c-Jun (A), and CARP-1 (B) proteins were determined in the cells treated with WA for $12 \mathrm{~h}$. In addition, MPM cells were either untreated (DMSO, denoted as 0 ) or treated with $10 \mu \mathrm{M}$ WA for the indicated time periods, and the levels of CARP-1, vimentin (C), and phospho-p38 (D) were determined by western blotting. The membranes in panels A-C were subsequently analyzed for levels of actin to assess protein loading, while the membrane in panel $D$ was probed for expression of total $p 38$.

doi:10.1371/journal.pone.0041214.g004

treatments stimulated CARP-1 expression in MPM cells (Fig. 4B, C), we next investigated whether WA activated p38, and the extent CARP-1 was required in transducing growth inhibitory effects of WA. Exposure of MPM cells to WA resulted in activation (phosphorylation) of p38 as early as $2 \mathrm{~h}$ while robust p38 activation was noticeable at the $6 \mathrm{~h}$ and subsequent periods of treatment (Fig. 4D). The extent CARP-1 was required for MPM cell growth inhibition by WA was determined next by experiments involving siRNA-dependent knock-down of CARP-1. H2461 cells were transfected with $100 \mathrm{nM}$ of each of the scrambled or CARP1 siRNAs essentially as described before [21] for 72 hours and the cells were then either untreated or treated with WA. Western blot analysis of the cell lysates revealed that CARP-1 siRNAs, but not scrambled siRNAs, caused loss of CARP-1 expression (Fig. 5A). Although WA stimulated CARP-1 expression in scrambled siRNA-transfected MPM cells, it failed to increase CARP-1 levels in cells transfected with CARP-1 siRNAs (Fig. 5A). In a separate experiment, human and murine MPM cells were similarly transfected with CARP-1 or scrambled siRNAs followed by their treatment with WA essentially as in Fig. 5A, and cell viabilities were determined by the MTT assay as in methods. WA inhibited growth of MPM cells transfected with scrambled siRNAs, whereas CARP-1 knock-down interfered with WA-dependent suppression of MPM cell growth (Fig. 5B). The data in Figs. 4 and 5 suggest that MPM inhibitory signaling by WA involves down-regulation of oncogenes c-myc and c-jun, activation of pro-apoptotic p38 kinase, and stimulation of CARP-1, while depletion of CARP-1 interferes with WA-dependent MPM cell growth inhibition.
WA inhibits tumor growth in mesothelioma allograft

To investigate whether WA inhibits MPM tumor growth, we implanted murine mesothelioma $\mathrm{AB} 12$ cells $\left(0.5 \times 10^{6}\right)$ subcutaneously into the right flanks of female Balb/c mice. When the tumors became palpable $\left(\sim 120 \mathrm{~mm}^{3}\right)$, mice were randomized into the 2 groups for treatment with vehicle control or WA. Doses of 2$5 \mathrm{mg} / \mathrm{kg}$ of WA were previously found to be efficacious without significant side effects in animal studies involving xenografts of human breast and prostate cancer cells as well as soft tissue sarcomas $(12,13)$. Accordingly, the mice bearing AB12 cellderived tumors were administered with $5 \mathrm{mg}$ of WA/ $\mathrm{kg}$ of body weight given every day i.p. for 17 days. Inhibition of tumor growth by WA was observed after a 17 day-treatment, indicating the efficacy of WA against mesothelioma (Fig. 6A). After 17 days treatment, control tumors grew to an average size of $1774 \mathrm{~mm}^{3}$, and WA-treated tumors grew to $714 \mathrm{~mm}^{3}$, corresponding to a $60 \%$ inhibition (Fig. 6A). Tumor weights were measured by the end of the experiment. The average of tumor weight in vehicle treated group was $1.42 \mathrm{~g}$, while it was $0.46 \mathrm{~g}$ in WA-treated group, showing a $68 \%$ reduction in tumor weight (data not shown). More importantly, we found absence of any tumor in one mouse in the WA-treated group underscoring a complete response (data not included).

To determine whether WA suppressed murine MPM tumor growth by attenuating proteasome activity in vivo, we extracted proteins from the tumor remnants and used them for multiple assays as below. We found that the proteasomal chymotrypsin-like activity was inhibited by $25 \%$ in the mesothelioma tumors from mice treated with WA compared to vehicle-treated mice (Fig. 6B). This was associated with the accumulation of ubiquitinated 
Table 1. List of select WA-regulated genes in H2373 MPM cells.

\begin{tabular}{|c|c|c|c|c|c|}
\hline ID & SYMBOL & Name & ENTREZ & FoldChange & Direction \\
\hline 6380717 & HSPA1A & heat shock $70 \mathrm{kDa}$ protein $1 \mathrm{~A}$ & 3303 & 26.526557 & DOWN \\
\hline 5670465 & ADM & adrenomedullin & 133 & 25.887796 & DOWN \\
\hline 3850433 & HSPA1B & heat shock $70 \mathrm{kDa}$ protein $1 \mathrm{~B}$ & 3304 & 24.628061 & DOWN \\
\hline 60079 & DNAJB1 & $\begin{array}{l}\text { DnaJ (Hsp40) homolog, subfamily B, } \\
\text { member } 1\end{array}$ & 3337 & 24.051914 & DOWN \\
\hline 870379 & ZFAND2A & zinc finger, AN1-type domain $2 \mathrm{~A}$ & 90637 & 15.772064 & DOWN \\
\hline 1340600 & PPP1R15A & $\begin{array}{l}\text { protein phosphatase } 1 \text {, regulatory } \\
\text { (inhibitor) subunit } 15 \mathrm{~A}\end{array}$ & 23645 & 11.242614 & DOWN \\
\hline 6660601 & HMOX1 & heme oxygenase (decycling) 1 & 3162 & 8.7309379 & DOWN \\
\hline 160092 & HSPA6 & heat shock $70 \mathrm{kDa}$ protein 6 (HSP70B') & 3310 & 8.2488636 & DOWN \\
\hline 830619 & DDIT3 & DNA-damage-inducible transcript 3 & 1649 & 7.4905684 & DOWN \\
\hline 6860377 & DUSP1 & dual specificity phosphatase 1 & 1843 & 6.8823963 & DOWN \\
\hline 4610189 & HERPUD1 & $\begin{array}{l}\text { homocysteine-inducible, endoplasmic } \\
\text { reticulum stress-inducible, ubiquitin-like } \\
\text { domain member } 1\end{array}$ & 9709 & 5.7754888 & DOWN \\
\hline 5420095 & MYC & $\begin{array}{l}\text { v-myc myelocytomatosis viral oncogene } \\
\text { homolog (avian) }\end{array}$ & 4609 & 5.2859313 & DOWN \\
\hline 150327 & HSPH1 & heat shock $105 \mathrm{kDa} / 110 \mathrm{kDa}$ protein 1 & 10808 & 5.0773806 & DOWN \\
\hline 4390450 & SGK1 & serum/glucocorticoid regulated kinase 1 & 6446 & 5.0676214 & DOWN \\
\hline 4880673 & GADD45A & $\begin{array}{l}\text { growth arrest and DNA-damage-inducible, } \\
\text { alpha }\end{array}$ & 1647 & 4.8698733 & DOWN \\
\hline 7570324 & ID3 & $\begin{array}{l}\text { inhibitor of DNA binding 3, dominant } \\
\text { negative helix-loop-helix protein }\end{array}$ & 3399 & 4.6867504 & DOWN \\
\hline 870338 & EGR1 & early growth response 1 & 1958 & 4.3381067 & DOWN \\
\hline 7160239 & FOSB & $\begin{array}{l}\text { FBJ murine osteosarcoma viral oncogene } \\
\text { homolog B }\end{array}$ & 2354 & 4.2438425 & DOWN \\
\hline 6510367 & JUN & jun oncogene & 3725 & 3.972581 & DOWN \\
\hline 1710553 & HSPA6 & heat shock 70 kDa protein 6 (HSP70B') & 3310 & 3.6847097 & DOWN \\
\hline 1340075 & BAG3 & BCL2-associated athanogene 3 & 9531 & 3.6837105 & DOWN \\
\hline 4210524 & RND3 & Rho family GTPase 3 & 390 & 3.4998418 & DOWN \\
\hline 730286 & TXNRD1 & thioredoxin reductase 1 & 7296 & 3.4690154 & UP \\
\hline 1260086 & ID2 & $\begin{array}{l}\text { inhibitor of DNA binding 2, dominant } \\
\text { negative helix-loop-helix protein }\end{array}$ & 3398 & 3.2341928 & DOWN \\
\hline 4280017 & FOS & $\begin{array}{l}\mathrm{v} \text {-fos FBJ murine osteosarcoma viral } \\
\text { oncogene homolog }\end{array}$ & 2353 & 3.1238524 & DOWN \\
\hline 4920110 & GADD45B & $\begin{array}{l}\text { growth arrest and DNA-damage-inducible, } \\
\text { beta }\end{array}$ & 4616 & 3.0608382 & DOWN \\
\hline 4860286 & UBB & ubiquitin $B$ & 7314 & 2.7247999 & DOWN \\
\hline 670386 & ID1 & $\begin{array}{l}\text { inhibitor of DNA binding 1, dominant } \\
\text { negative helix-loop-helix protein }\end{array}$ & 3397 & 2.7209816 & DOWN \\
\hline 7650358 & TGFBI & $\begin{array}{l}\text { transforming growth factor, beta- } \\
\text { induced, } 68 \mathrm{kDa}\end{array}$ & 7045 & 2.3790355 & UP \\
\hline 1170709 & CDH2 & $\begin{array}{l}\text { cadherin 2, type 1, N-cadherin } \\
\text { (neuronal) }\end{array}$ & 1000 & 2.3726027 & UP \\
\hline 780270 & TIMP2 & TIMP metallopeptidase inhibitor 2 & 7077 & 2.2000615 & UP \\
\hline 4040097 & KLF6 & Kruppel-like factor 6 & 1316 & 2.1886976 & DOWN \\
\hline 3190148 & DDIT4 & DNA-damage-inducible transcript 4 & 54541 & 2.1817718 & DOWN \\
\hline 830661 & DCBLD2 & $\begin{array}{l}\text { discoidin, CUB and LCCL domain } \\
\text { containing } 2\end{array}$ & 131566 & 2.1806644 & UP \\
\hline 7050278 & RUNX1 & runt-related transcription factor 1 & 861 & 2.1623177 & DOWN \\
\hline 3710544 & EIF1 & eukaryotic translation initiation factor 1 & 10209 & 2.1583103 & DOWN \\
\hline 670086 & MXD1 & MAX dimerization protein 1 & 4084 & 2.1341046 & DOWN \\
\hline 1470215 & MAP3K8 & $\begin{array}{l}\text { mitogen-activated protein kinase kinase } \\
\text { kinase } 8\end{array}$ & 1326 & 2.1100298 & DOWN \\
\hline
\end{tabular}


Table 1. Cont.

\begin{tabular}{llllll}
\hline ID & SYMBOL & Name & ENTREZ & FoldChange & Direction \\
\hline 3060300 & UBC & ubiquitin C & 7316 & 2.0749465 & DOWN \\
6660343 & RBM14 & RNA binding motif protein 14 & 10432 & 2.0674976 & DOWN \\
\hline
\end{tabular}

Genes upregulated by WA are in bold.

doi:10.1371/journal.pone.0041214.t001

proteins and the proteasome target Bax (Fig. 6C). Increased expression of proteasome targets, p27 was determined following immunostaining of the treated and control tumors using anti-p27 antibodies. Elevated levels of p27 was evident in tumors from mice treated with WA (Fig. 6D), further confirming that WA was able to inhibit tumor proteasome activity in vivo. Further, levels of apoptosis in the control and WA-treated tumors were determined using the TUNEL assay, as well as immunostaining the tumors tissues for levels of c-myc and CARP-1 proteins. The TUNEL staining indicated an increase in the number of apoptotic cells in the tumors from mice treated with WA when compared with the corresponding vehicle-treated control (Fig. 6D). Similar to the in vitro studies, c-myc expression was diminished while CARP-1 levels were elevated in tumors derived from mice treated with WA. Taken together, these data show that WA has the ability to regress mesothelioma growth in vivo in part by inhibiting proteasome and stimulating apoptosis.

\section{Discussion}

WA is the major and the most active component of dietary supplement Ashwagandha. Recent preclinical studies have revealed that WA targets multiple molecules for mediating cell death in a variety of cancer cells. WA exhibited anti-angiogenesis effects through NF- $\kappa \mathrm{B}$ inhibition, and the ubiquitin-proteasome pathway was thought to be involved [32]. Our previous work has revealed direct inhibition on the proteasomal chymotryptic activity by WA, which likely contributes to cell death induction and tumor growth inhibition in human prostate cancer [14]. WA has also been reported to activate prostate apoptosis response-4 (Par-4) gene, which contributes to regression of PC-3 xenografts in nude mice [12]. WA treatment generated reactive oxygen species (ROS), causing cell death in HL-60 cells [18]. Consistent with these findings, we found that WA treatments increased pro-apoptotic protein Bax and NF- $\kappa \mathrm{B}$ inhibitory protein $\mathrm{I} \kappa \mathrm{B}-\alpha$ in the patient derived MPM cells. WA also decreased the proteasomal chymotryptic activity in human and murine MPM cells, suggesting that WA targets multiple pathways to suppress MPM growth. Cell proliferation and cell death are regulated by the balance between proapoptotic and antiapoptotic proteins [33]. Increased level of pro-apoptotic protein $\mathrm{Bax}$ and $\mathrm{I} \kappa \mathrm{B}-\alpha$ in the presence of WA (Figs. 2, 3) would make the human MPM cells switch to apoptotic cell death. Indeed, WA treatment induced caspase-3 activation, PARP cleavage, and morphological changes (condensed nucleus) characteristic of apoptosis in MPM cells (Figs. 2, 3) strongly underscoring its pro-apoptotic properties.

Simultaneous blocking of cancer cell growth and survival pathways while activating apoptosis is a powerful approach to effectively suppress cancer. Our current studies indicating involvement of apoptosis in WA-dependent inhibition of MPM growth highlights anti-MPM potential for this safe and non-toxic agent. In this context, our studies are consistent with a number of prior studies demonstrating involvement of apoptosis signaling in WA-dependent suppression of human breast and prostate cancer cell growth as well as the soft tissue sarcomas [11-13]. The fact that alterations in apoptosis signaling pathways often contribute to MPM growth and survival [34], we undertook an in vitro and gene-array based profiling to identify novel transducers of

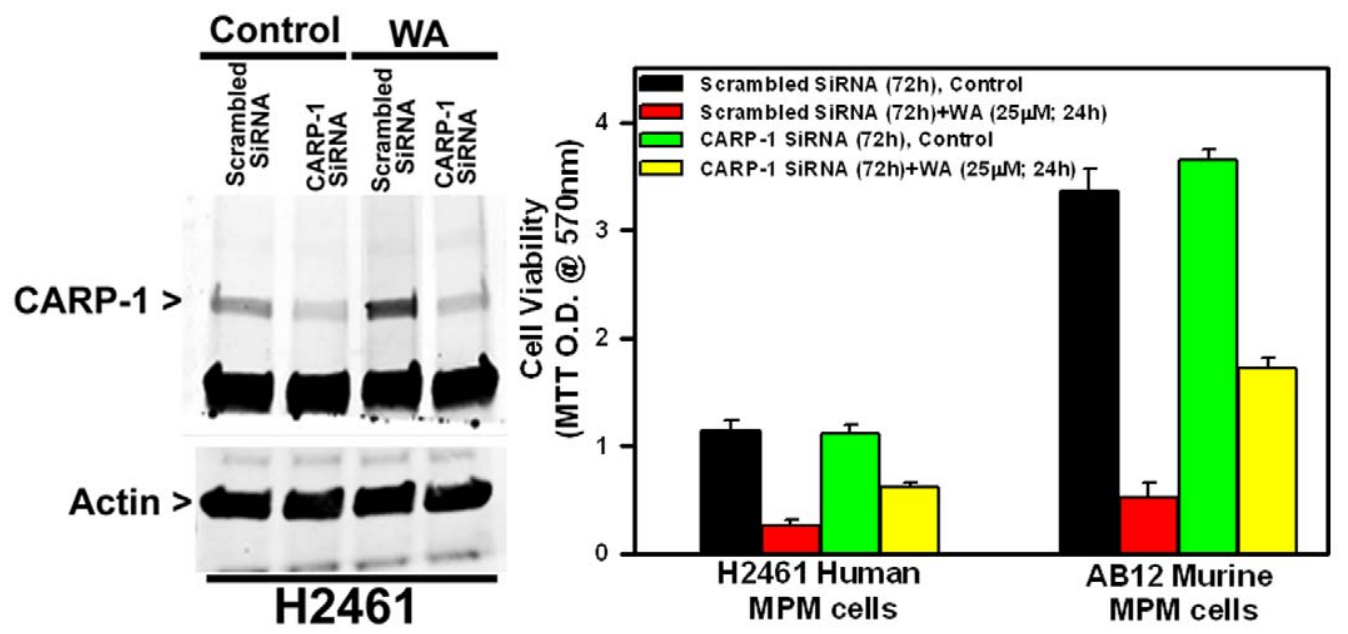

Figure 5. CARP-1 is required for MPM cell growth inhibition by WA. Knockdown of CARP-1 blocks WA effects. Cells were transfected with $100 \mathrm{nM}$ each of scrambled or CARP-1 siRNAs for $72 \mathrm{~h}$ and then were either untreated (Control, DMSO) or treated with $10 \mu \mathrm{M}$ WA for further $24 \mathrm{~h}$. Cell lysates were subjected to western blotting as in Fig. 4 for levels of CARP-1 and actin proteins or subjected to MTT assay for determination of cell viabilities essentially as in Fig. 1. Columns in B represent means of three independent experiments; bars, S.E. * and \#, $p=, 0.01$ relative to WA-treated, scrambled siRNA-transfected cells.

doi:10.1371/journal.pone.0041214.g005 


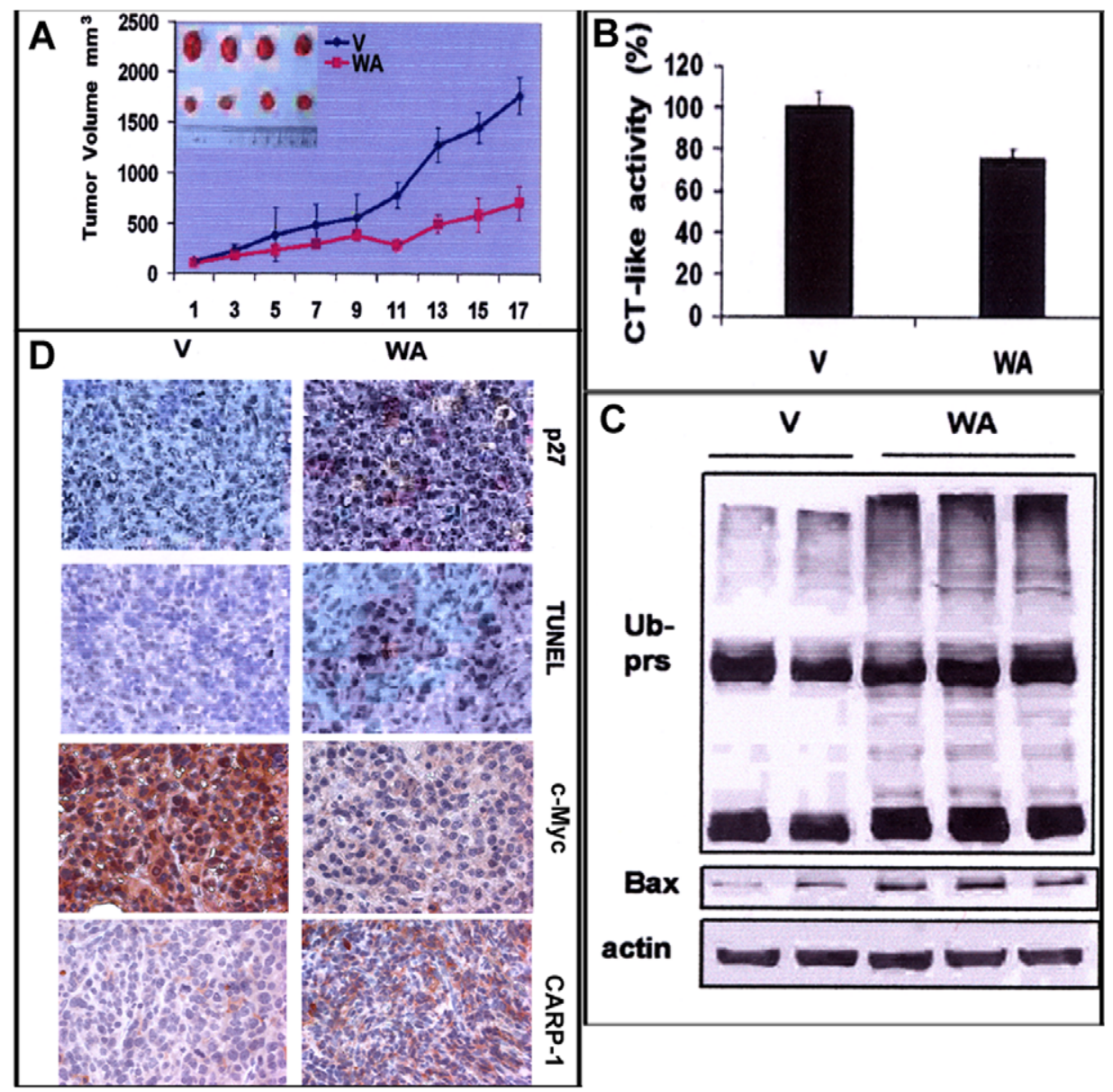

Figure 6. WA inhibited tumor growth in murine MPM allograft. Female BALB/c mice bearing AB 12 tumors were treated with either vehicle (V) or WA as described in methods. Animals were monitored every day for tumor volumes (growth) as well as any activity alteration. Tumor volumes were measured every other day for the control and treated groups (A). Points, mean tumor volume in each experimental group containing 4 mice. Bars, SD. Tumors were collected after 17 days of treatment, and the tumor biopsies were analyzed by the proteasomal chymotrypsin-like activity assay $(B)$ and Western blotting for ubiquitinated proteins (Ub-prs) and Bax $(C)$ essentially as detailed in methods. D, The tumor tissues from the control and treated groups were immuno-stained for apoptosis (by TUNEL assay) and levels of CDKI p27, oncogene c-myc and CARP-1 proteins as noted in methods. Magnifications are $400 \times$.

doi:10.1371/journal.pone.0041214.g006

apoptosis signaling that may be activated/induced by WA. Our studies revealed that while WA suppressed levels of several growthpromoting genes it also caused elevated expression of cell growth inhibitory and apoptosis transducers such as CCAR1/CARP-1, and TIMP2. Our gene-array-based analyses (table 1) together with western blot data in figs. 4 and 5 convincingly reveal a new group of apoptosis signal transducing genes that are activated by WA in MPM cells in vitro and in vivo. Since, GCAR1/CARP-1 is an emerging and novel target of diverse cell growth and apoptosis signaling pathways [19-21] and is not involved in cisplatindependent HBC growth inhibition [24], identification of CGAR1/ CARP-1 as a downstream effector of WA signaling would be useful for design of effective strategies for optimizing WA potential as suppressor of MPM and its drug (Cisplatin)-resistant phenotype.

The gene array data also revealed a number of interesting genes that may be involved in regulating MPM cell growth and survival in the presence of WA. Of note is the down regulation of oncogene c-myc, and AP-1 transcription factor components c-fos and c-jun (table 1). Since c-myc and AP-1 signaling are down-stream of the growth and metastasis-promoting Ras-Raf-Erk pathway, whether and to the extent WA targets upstream regulators of this pathway to block MPM growth and metastases remains to be clarified. WA treatments however resulted in activation of pro-apoptotic p38 MAPK/SAPK that is often known to transduce stress-dependent inhibitory signals. Moreover, WA not only suppressed levels of the tumorigenesis and metastasis-associated vimentin protein, it also caused elevated levels of cancer cell inhibitory, extra cellular matrix-associated TIMP2 protein. Thus the mechanism of action of WA would appear to involve pleiotropic effects including (a) activation of signal transduction pathways, (b) stimulation of apoptosis, (c) blockage of proteasome, and (d) inhibition of growth and metastasis-promoting genes such as c-myc and vimentin.

WA exerted potent inhibition on the growth of mesothelioma AB12 allografts. Consistent with the anti-MPM effects of WA in vitro, inhibition of proteasome also correlated with cell death induction in vivo. Treatment of tumors with WA, but not vehicle, caused inhibition of proteasomal chemotrypsin-like activity and accumulation of its substrate Bax as well as ubiquitinated proteins (Fig. 6). The proteasome inhibition also resulted in extensive accumulation of p27 in situ in WA-treated tumors (Fig. 6D). The WA-treated tumors also elicited TUNEL positivity and morphological features associated with apoptosis, demonstrating that 
proteasome inhibition by WA also triggers apoptosis in vivo (Fig. 6D). Finally, since WA treatments caused regression of murine MPM tumor growth (Fig. 6A) to the extent that a complete regression of tumor growth was in noted in one WA-treated animal, suggests that WA can reach a therapeutic concentration in vivo to facilitate its direct targeting and inhibition of tumor cellular proteasome, resulting in elevated apoptosis induction and tumor growth inhibition.

In brief, the data presented here convincingly demonstrate that WA targets multiple pathways to suppress MPM growth in vitro and in vivo, and underscore its potential as a future anti-MPM therapy.

\section{References}

1. Kamp DW (2009) Asbestos-induced lung diseases: an update. Transl Res 153(4):143-52

2. Su S (2009) Mesothelioma: path to multimodality treatment. Semin Thorac Cardiovasc Surg 21(2):125-31.

3. Tsao AS, Wistuba I, Roth JA, Kindler HL (2009) Malignant pleural mesothelioma. J Clin Oncol 27(12):2081-90.

4. Peto J, Decarli A, La Vecchia G, Levi F, Negri E (1999) The European mesothelioma epidemic. Br J Cancer 79(3-4):666-72.

5. Wang Y, Rishi AK, Puliyappadamba VT, Sharma S, Yang H et al. (2010) Targeted proteasome inhibition by Velcade induces apoptosis in human mesothelioma and breast cancer cell lines. Cancer Chemother Pharmacol 66: 455-466.

6. Dou QP, Smith DM, Daniel KG, Kazi A (2003) Interruption of tumor cell cycle progression through proteasome inhibition: implications for cancer therapy. Progress in cell cycle research 5:441-6.

7. Yang H, Landis-Piwowar KR, Chen D, Milacic V, Dou QP (2008) Natural compounds with proteasome inhibitory activity for cancer prevention and treatment. Curr Protein Pept Sci 9(3):227-39.

8. Devi PU (1996) Withania somnifera Dunal (Ashwagandha): potential plant source of a promising drug for cancer therapy and radiosensitization. Ind J Exp Biol 34: 927-932.

9. Chowdhury K, Neogy RK (1975) Mode of action of Withaferin A and Withanolide D. Biochem Pharmacol 24: 919-920.

10. Bhattacharya SK, Bhattacharya D, Sairam K, Ghosal S (2002) Effect of Withanis somnifera glycowithanolides on a rat model of tardive dyskinesia. Phytomedicine 9: 167-170.

11. Stan SD, Hahm ER, Warin R, Singh SV (2008) Withaferin A causes FOXO3aand Bim-dependent apoptosis and inhibits growth of human breast cancer cells in vivo. Cancer Res 68: 7661-7669.

12. Srinivasan S, Ranga RS, Burikhanov R, Han SS, Chendil D (2007) Par-4dependent apoptosis by the dietary compound withaferin A in prostate cancer cells. Cancer Res 67: 246-253.

13. Lahat G, Zhu Q-S, Huang K-L, Wang S, Bolshakov S, et al. (2010) Vimentin is a novel anti-cancer therapeutic target; insights from in vitro and in vivo mice xenograft studies. PLoS One 5(4) e10105.

14. Yang H, Shi G, Dou QP (2007) The tumor proteasome is a primary target for the natural anticancer compound Withaferin A isolated for "Indian winter cherry". Mol Pharmacol 71: 426-437.

15. Oh JH, Lee TJ, Park JW, Kwon TK (2008) Withaferin A inhibits iNOS expression and nitric oxide production by Akt inactivation and down-regulating LPS-induced activity of NF-kappaB in RAW 264.7 cells. Eur J Pharmacol 599: 11-17.

16. Malara N, Foca D, Casadonte F, Sesto MF, Macrina L, et al. (2008) Simultaneous inhibition of the constitutively activated nuclear factor kappaB and of the interleukin- 6 pathways is necessary and sufficient to completely overcome apoptosis resistance of human U266 myeloma cells. Cell Cycle 7: 3235-3245.

17. Falsey RR, Marron MT, Gunaherath GM, Shirahatti N, Mahadevan D, et al. (2006) Actin microfilament aggregation induced by withaferin A is mediated by annexin II. Nat Chem Biol 2: 33-38.

18. Malik F, Kumar A, Bhushan S, Khan S, Bhatia A, et al. (2007) Reactive oxygen species generation and mitochondrial dysfunction in the apoptotic cell death of

\section{Supporting Information}

Table S1 List of WA-regulated genes in H2373 MPM cells.

(XLS)

\section{Author Contributions}

Conceived and designed the experiments: AW AKR QPD. Performed the experiments: HY YW VTC WW CQC LAP. Contributed reagents/ materials/analysis tools: HIP. Wrote the paper: AW AKR QPD.

human myeloid leukemia HL-60 cells by a dietary compound withaferin A with concomitant protection by N-acetyl cysteine. Apoptosis 12: 2115-2133.

19. Rishi AK, Zhang L, Boyanapalli M, Wali A, Mohammad RM, et al. (2003) Identification and characterization of a Cell-Cycle and Apoptosis Regulatory Protein (CARP)-1 as a novel mediator of apoptosis signaling by Retinoid CDD37. J Biol Chem 278: 33422-33435.

20. Rishi AK, Zhang L, Yu Y, Jiang Y, Nautiyal J, et al. (2006) Cell cycle and apoptosis regulatory protein (CARP)-1 is involved in apoptosis signaling by epidermal growth factor receptor. J Biol Chem, 281(19):13188-98.

21. Puliyappadamba VT, Wu W, Bevis D, Zhang L, Polin L, et al. (2011) Antagonists of anaphase promoting complex (APC)-2-cell cycle and apoptosis regulatory protein (CARP)-1 interaction are novel regulators of cell growth and apoptosis. J Biol Chem 286 (44): 38000-38017.

22. Pass HI, Lott D, Lonardo F, Harbut M, Liu Z, et al. (2005) Asbestos exposure, pleural mesothelioma, and serum osteopontin levels. N Engl J Med 353(15): 1564-73.

23. Suzuki E, Kim S, Cheung HK, Corbley MJ, Zhang X, et al. (2007) A novel small-molecule inhibitor of transforming growth factor beta type I receptor kinase (SM16) inhibits murine mesothelioma tumor growth in vivo and prevents tumor recurrence after surgical resection. Cancer Res 67(5):2351-9).

24. Odaka M, Sterman D, Wiewrodt R, Zhang Y, Kiefer M, et al. (2001) Eradication of intraperitoneal and distant tumor by adenovirusmediated interferon-b gene therapy due to induction of systemic immunity. Cancer Res 61:6201-12.

25. Smyth GK, Limma L (2005) Liner models for microarray data. In: Bioinformatics and Computational Biology Solutions Using R and Bioconductor, edited by Gentleman R, Carey V, Duoit S, Irizarry R, Huber W. New York: Springer, p. 397-420.

26. Tarca AL, Carey VJ, Chen XW, Romero R, Drãghici S (2007) Machine learning and its applications to biology. PLoS Comput Biol 3: e116.

27. Zhang L, Levi E, Majumder P, Yu Y, Aboukameel A, et al. (2007) Transactivator of transcription tagged cell cycle and apoptosis regulatory protein-1 peptides suppress growth of human breast cancer cells in vitro and in vivo. Mol Cancer Ther 6(5): 1661-1672.

28. Kim JH, Yang CK, Heo K, Roeder RG, An W, et al. (2008) CCAR1, a key regulator of mediator complex recruitment to nuclear receptor transcription complexes. Mol Cell 31, 510-519.

29. Wang Y, Rishi AK, Wu W, Polin L, Sharma S, et al. (2011) Curcumin suppresses growth of mesothelioma cells in vitro and in vivo, in part, by stimulating apoptosis. Molecular \& Cellular Biochemistry 357: 83-94.

30. Kalluri R, Weinberg RA (2009) The basics of epithelial-mesenchymal transition. J Clin Invest 119: 1420-1428.

31. Bargagna-Mohan P, Hamza A, Kim YE, Khuan Abby Ho Y, Mor-Vaknin N, et al. (2007) The tumor inhibitor and antiangiogenic agent withaferin A targets the intermediate filament protein vimentin. Chem Biol 14: 623-634.

32. Mohan R, Hammers HJ, Bargagna-Mohan P, Zhan XH, Herbstritt CJ, et al. (2004) Withaferin A is a potent inhibitor of angiogenesis. Angiogenesis 7: 115122.

33. Jolly C, Morimoto RI (2000) Role of the heat shock response and molecular chaperones in oncogenesis and cell death. J Natl Cancer Inst 92(19):1564-72.

34. Gordon GJ, Mani M, Mukhopadhyay L, Dong L, Yeap BY, et al. (2007) Inhibitor of apoptosis proteins are regulated by tumour necrosis factor-alpha in malignant pleural mesothelioma. J Pathol 211(4):439-46. 